\title{
Agent-based Three Layer Framework of Assembly-Oriented Planning and Scheduling for Discrete Manufacturing Enterprises
}

\author{
Yinghui Fan, Sohel Anwar, Litao Wang
}

\begin{abstract}
To solve the cost burden caused by delivery tardiness for small and medium sized packaging machinery enterprises, the assembly-oriented planning and scheduling is studied based on the multi-agent technology. Taking into account the due date, the planning and scheduling are optimized iteratively with the rule-based algorithms complying with the machining and assembling process constraints. To make the realistic large-scale production planning scheme tailored to fit the optimization algorithms, a multi-agent system is utilized to conceptually construct a three-layer framework corresponding to three planning horizons of different task level. These planning horizons are quarter/month of product/subassembly level, week of part level, and day of operation level. With the strategy of combining top-down task decomposition and bottom-up plan update (where the quarterly orders are decomposed into the monthly, weekly, and daily tasks according to the product processing structure while the resulting plans of every layer are updated iteratively based on the bottom layer optimization), the proposed framework not only addresses the planning but also the periodic and event-driven scheduling of the processes. In this paper, a gravure printing machine is considered as a test case for evaluating the proposed framework. The simulation results with the historical data of the orders for this machine demonstrate the effectiveness of the proposed scheme on the control of the distribution of idle-time. It can also provide a resolution to tardiness penalty for small and medium sized enterprises.
\end{abstract}

\section{INTRODUCTION}

Packaging machinery enterprise is one of the discrete manufacturing industries characterized by multi-species, low-volume, and build-to-order. Most of them are of small-and-medium size with less ability to address the fluctuations over the periods of boom-and-bust. Moreover, without proper connection between the machining shop and assembly shop, order turnaround times will be negatively impacted for assembly procedures which may have to be halted due to a lack of components during peak demand periods. The resulting delays mean higher costs. This paper attempts to control the tardy time with the self-developed Production Management System (PMS) based on the multi-agent technology.

Yinghui Fan is with Department of Mechanical Engineering, Purdue School of Engineering and Technologies, IUPUI Purdue University, Indianapolis, IN 46202 USA, on leave from Shantou University, Shantou, GD 515063 China. (e-mail: yingfan@iupui.edu).

Sohel Anwar is with Department of Mechanical Engineering, Purdue School of Engineering and Technologies, IUPUI Purdue University, Indianapolis, IN 46202 USA. (corresponding author, phone: 317-274-7640; fax: 317-274-9744; e-mail: soanwar@ iupui.edu).

Litao Wang is with the Electromechanical Engineering Department, Shantou University, Shantou, GD 515063 China (e-mail: 151twang@stu.edu.cn).
Multi-Agent system (MAS) has been shown to be effective to accommodate complex manufacturing environment [1] [2]. Taking advantage of the distributed structure, the complicated planning problem can be decomposed into more easily solvable sub-problems. The demand of flexibility, agility, and reconfiguration ability of production systems can be well met with the intelligent mechanism of MAS. Thus, it is widely exploited to optimize cooperation of manufacturing enterprises, production planning and scheduling, and floor shop control [3].

For hybrid flow shop scheduling problems, Luo et al. [4] proposed two-step model and a genetic algorithm was used to solve it combining with clustering algorithm in the condition of a set of complicated rules and constraints. For distributed and dynamic manufacturing environment, Wang and Lin [5] developed an agile manufacturing planning and control system with the characteristics of visibility, accountability, track ability, responsiveness, and flexibility by the integration of RFID technique and MAS. Similarly, for stochastic production of mold industry, Liu et al. [6] proposed a framework based on MAS to provide a reliable and competitive due date quotation significant for delivery reliability performance. With a knowledge-based multi-agent evolutionary algorithm (KMEA) presented in [7], Wang et al. gave a solution to semiconductor final testing scheduling problem and demonstrated the effectiveness and efficiency of KMEA with numerical simulations.

In spite of the huge amount of reported works on the research of MAS and its application, the generic design methods are still very limited and the development of agent-based production system is rather case-dependent [8]. This paper aims at optimizing the assembly-oriented planning and scheduling of packaging machinery enterprises with multi-agent architecture to solve the delivery tardiness. Firstly, in addition to machining processes, assembly processes are considered simultaneously as planning constraints to guarantee on-demand delivery. Secondly, for the assembly-oriented planning characterized by large-scale data is NP-hard (non-deterministic polynomial-time) and difficult to utilize most optimizing algorithms directly, a three-layer framework corresponding to three planning horizons is constructed with cooperative agents combining top-down task decomposition and bottom-up plan update. By top-down task decomposition strategy, the quarterly tasks can be divided into the monthly, weekly, and daily ones based on the processing structure of products. The developed rules and algorithms are applied to optimize the planning of every layer; furthermore, the resulting plans are updated with the bottom layer optimization till the tardiness can be resolved to a satisfactory level, which means the acceptable balance is reached between tardy costs (sum of tardiness penalties and additional warehouse cost) and outsourcing costs. 
The paper is arranged as follows: the proposed agent-based three-layer framework is introduced in Section II and the detailed scheduling model for each layer is described focusing on heuristic rules. Section III presents the simulation of planning and scheduling processes based on the proposed framework with historical data and its effectiveness is discussed by comparing with empirical scheduling. Finally, the conclusion is summarized in section IV.

\section{AGENT-BASED THREE-LAYER FRAMEWORK}

The realistic floor shop scheduling problem is rather challenging due to its characteristics of large scale data, complex constraints, and heterogeneous environment. Any single optimization method or algorithm is unlikely to be sufficient for such scheduling. From this perspective, an agent-based floor shop scheduling model — a three-layer framework taking advantage of MAS distributed structure is proposed as Figure 1, which corresponds to three planning horizons, i.e., quarter/month, week, and day. According to the machining and assembly processes, the massive quarterly task (Usually the contracted orders are managed by quarter in this kind of machinery factory) can be decomposed into weekly and daily sub-tasks layer by layer under the guideline of matching task hours with production capacity. This top-down decomposition makes it much easier for weekly and daily task agents to choose appropriate optimization algorithm, and the local optimal weekly and daily plans are employed to update upward and tardy condition will be checked up accordingly. The decomposition and update will be implemented iteratively till no tardiness exists or tardy costs are balanced against outsourcing costs to an acceptable degree, i.e. the sub-global optimization is achieved.

The strategy of top-down decomposition and bottom-up update is not only capable of planning, but also adaptable to periodic adjustments by monitoring the plan implementation, and event-driven adjustments in the case of order changes, machine breakdowns, and worker changes etc. Thus, it is worth pointing out that the proposed three-layer framework can address both planning and dynamic scheduling. The execution state can be updated timely through the data collection and analysis at quality testing stage of every process. Meanwhile, the resource agent is on the alert for any incidental events of machines and workers.

As one can see, with the use of three-layer framework, PMS can fulfill the realistic monitoring and control for the shop floor production. Within the framework, the task decomposition and plan update are implemented depending on the autonomy of every agent and interactive cooperation amongst agents of same layer and the negotiation among those of different layers.

As shown in Figure 1, hybrid approach is used for agent encapsulation [2], in which physical entities are mapped into physical agents with reactive architecture, such as order agents (OA), product agents (PA), task agents (TA), and machine agents (MA), machining unit agent (MUA), etc., while system-level functional modules are encapsulated as deliberative agents, i.e., scheduling agent (SA) and resource agent (RA). The interaction among agents is proceeded by messages exchange with agent communication language, KQML [9], as illustrated in Figure 2.

To relieve the burden of data storage and exchange of every agent, a unified database is developed to manage related data, such as the orders, the product processing structure, and the resources, whereas only the corresponding database addresses are saved in the knowledge base of agents. When the sensor component of functional agent (FA) perceives the task instruction, such as sequencing or resource allocation, from the effector of physical entity agent (PEA), it will be handled by the analyzer and decision-maker based on its own rule library and knowledge base. This process is assisted by accessing database to get necessary data and update them simultaneously. Finally, the FA effector would tell the sensor of PEA what action has been done and which database address should be accessed to get the expected result.

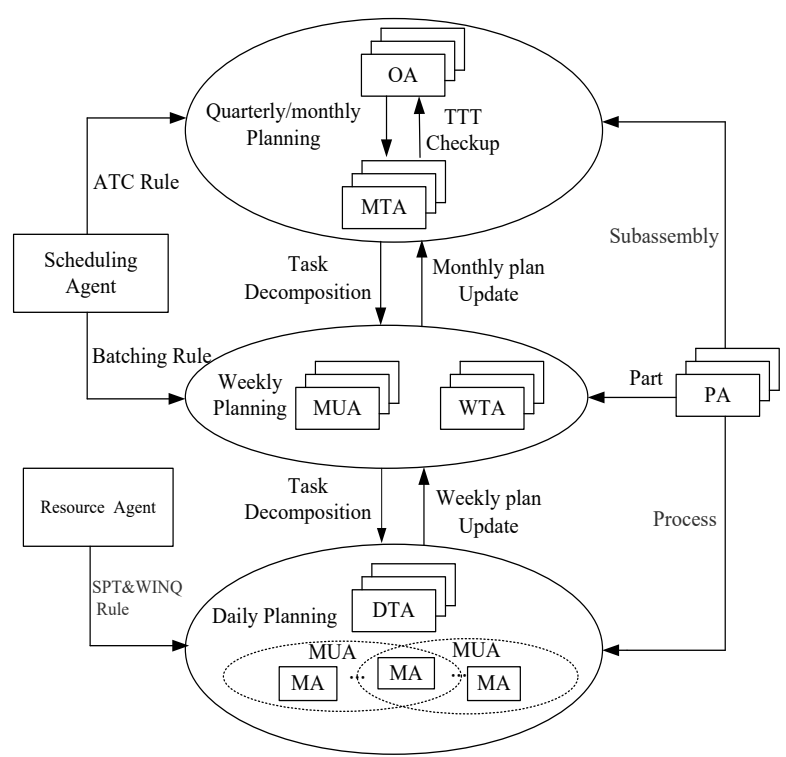

Figure 1. Agent-based three-layer framework

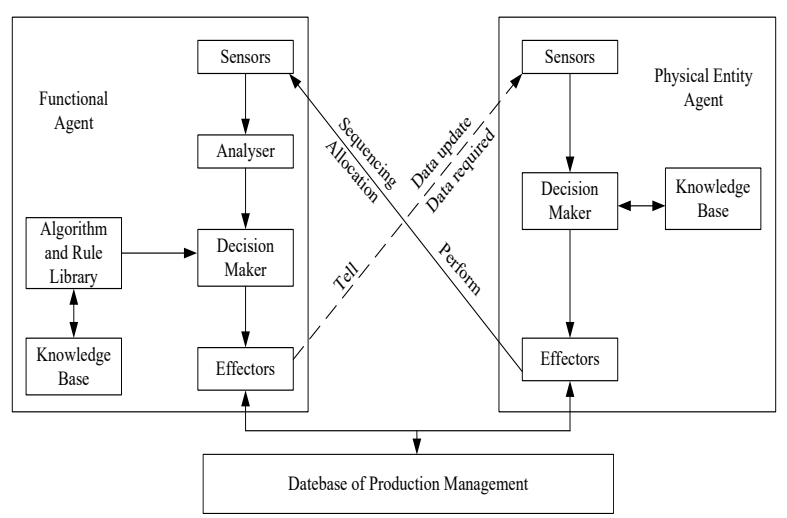

Figure 2. Agent architecture and communication

\section{A. Quarterly/monthly planning with ATC rule}

For quarterly planning, the sensor of OA of this quarter will firstly get the percept of the manufacturing task assignments. The weight of every order is decided by analytic hierarchy process (AHP) [10] based on the knowledge of due 
data urgency and customer level. The weighted orders are further decomposed into weighed products through the interaction with PAs. Finally, the decision-maker allows the effector of $\mathrm{OA}$ to send perform instruction of sequencing weighed products to the sensor of SA.

Priority rule of Apparent Tardiness Cost (ATC) [11] of the rule library permits $\mathrm{SA}$ to make the connection from percept to action, i.e., sequencing weighted products iteratively by the calculation of processing sequence index (PSI) and the product with the highest PSI chosen every time till all are scheduled. As a dynamic composite rule, ATC inherits the metrics of Weighted Shortest Processing Time (WSPT) and Minimum Slack (MS) with greedy heuristics, and extends them by a "look-ahead" feature through parameterization. The near- optimal solution can be achieved with minimizing weighted tardiness cost as the performance, and exhibiting robustness in most load conditions.

As shown in equation (1), the PSI of $j$ th $(j=1,2, \ldots N)$ of $N$ weighted products at the instance $t$ is computed based on its weight $w_{j}$, processing time $p_{j}$, due date $d_{j}$, and the average processing time $\bar{P}$ of all unscheduled weighted products. The exponential discounting is used to account the influence of slack on the maximum tardiness cost $w_{j} / p_{j}$. The looking-ahead parameter $K$ can be set as equation (2) when the due dates have the uniform distribution, i.e., taking into consideration only the influence of due date range factor $R$ and ignoring the tightness factor $\tau$ [12].

$$
\begin{aligned}
& I_{j}(t)=\frac{w_{j}}{p_{j}} \exp \left[-\frac{\max \left(d_{j}-p_{j}-t, 0\right)}{K \bar{P}}\right] \\
& \begin{cases}K=4.5+R, & R \leq 0.5 \\
K=6-2 R, & R>0.5\end{cases} \\
& R=\left(D_{\max }-D_{\min }\right) / C_{\max }
\end{aligned}
$$

The sequenced products constitute the quarterly plan, which is decomposed into corresponding monthly tasks by the rule of matching task working hours against shop production capacity (task-capacity-matching, TCM). Through communicating with PA based on the knowledge of SA, the effector will tell monthly task agents (MTA) of the monthly tasks in the form of subassembly. Meanwhile, the total tardy time (TTT) of quarterly orders is computed as:

$$
\begin{gathered}
T=\sum_{1}^{M} T_{i}=\sum_{1}^{M}\left[\max \left(0, C_{i}-D_{i}\right)\right] \\
i=1,2, \ldots, M, M \in[3,5]
\end{gathered}
$$

Where $T_{i}, C_{i}$ and $D_{i}$ are the tardy time, completion time and due date of the $i$ th order respectively.

In general, there are great similarities among subassemblies and their parts of packaging printing machine. For instance, the number of color printing units of gravure can be customized, but each of these units for certain gravure can be the same. Accordingly, the batching rules are employed to simplify the planning at the level of subassembly and parts [13]. With the assumption that their due dates decided by back forwarding rule and assembly constraints are consistent, the identical subassemblies among monthly tasks will be grouped together as a subassembly batch with the processing priorities of the highest among them, whereas there should be more concern for the batching period of the part batches of the same type parts. Thereafter, the resulting monthly plans will be decomposed downward into weekly tasks with the TCM rule and perceived by weekly task agents (WTA).

\section{B. Weekly planning with part batching rule}

Part batching is good for time-saving of setups and logistics cost reducing. Nevertheless, large batching size may lead to the idleness and the lower utilization of resources (machines and workers) due to the existence of processing constraints, which is detrimental for the balanced production. For the batching size is time period dependent, the key of Part batching (PB) rule is how to determine an appropriate batching period for weekly tasks to make feasible and cost-saving plan.

According to the field study, a feasible and proper weekly plan is one that does not need extra work hours beyond 9 $(3 \times 3$ overtime pattern). Thus, with the condition of resulting weekly plans being reasonable, the batching size should be maximized within the selected period. Accordingly, a proper batching period should be an integer value with the infimum as 1 day and the supremum 6 days. In other words, there are only 6 possible elements for the batching period set. To simplify the decision process, the planning with PB rule can be performed by selecting the batching period iteratively over the set in descending sequence till practicable weekly plans within one month resulted.

Furthermore, there are kinds of parts that require large work hours beyond 100 with the number of processes of over 50 ranging from 1 to 3 . Based on the field statistics, the percentage of large-work-hour (LWH) part is around 5\% and the average make-span is one to two weeks due to the processing constraint. In accordance with longest processing time (LPT) rule [14], the priorities of LWH parts should be adjusted higher and leave those of small-work-hour (SWH) parts with work hours less than 100 unchanged.

With the aid of PB rule and LPT rule implemented through the cooperation among WTA, SA and PA, the weekly plans can be determined. Subsequently, in line with TCM rule, the sequenced weekly job queue will be divided into segments with each assigned the corresponding resources, i.e. machining unit (MU) by the interaction of WTA, RA and MUA. Then, within every MU the queue can be further split into the daily task in the form of processes, which will be told to daily task agents (DTA).

\section{Daily planning with $G A$ and $M U$ dynamic reconfiguration}

For the quantity of jobs to be scheduled within MU in daily planning has been greatly reduced, genetic algorithm (GA) can be adopted to optimize the schedule with minimizing the maximum completion time of daily task as the objective function. The chromosome of operation-based representation is made up of operations sequencing of job and allocated machines, while the initial population size is around 100. For every iteration, the roulette wheel method is used in the selection step, and precedence operation crossover (POX) probability set as 0.8 and mutation probability as 0.1 to produce the new generation. The generational process will terminate if the make span is not shortened any more for the last two generations or the number of generation up to 20 . 
In general, the set of active schedules is frequently much smaller than that of all feasible schedules and contains a subset of schedules to which all optimal schedules must be equivalent [15]. With this in mind, we adopt the decoding algorithm with greedy insertion in the decoding process to produce active schedule [16], in which the jobs can be scheduled at their earliest feasible time in the assigned machine. However, there may be no time slot available to schedule operation for certain machine within the allocated MU for two reasons [13]. One is that the machine requested belongs to the bottleneck set, which can only be attached to certain MU at the processing interval on demand of DTA and intermediated by RA through negotiating with MA and MUA. The other is the required machine is overload due to the processes constraint though the capabilities of MU in total work hours is consistent with daily task according to TCM rule. In this case, the same type machine or the machine capable of performing the asked operation of other MUs needs to be evaluated by RA to confirm whether it can be assigned to this MU temporarily or not.

This dynamic reconfiguration of MU can be implemented with the combination rule of SPT/WINQ [17]. WINQ (Least Work In Next Queue) is a kind of assignment rule directed at balancing machine workload to implement the concept that it is when the idle-time occurs can be determined though the amount of idle-time cannot be affected by priority assignment [18]. By looking at the sum of the processing times of the same operation of the same type part of remaining daily task queues, the available machine will be assigned to certain MU corresponding to the queue with the shortest. Meanwhile, SPT (Shortest Processing Time) can also achieve satisfactory performance on the aspect of machine utilization as a rule for operation sequencing. The combination rule considers the amount of work remaining as well as the current processing time, and allows the weighting coefficient to vary dynamically according to the function of current shop balance. With this metric, SPT/WINQ is of great value to ensure the utilization in the decoding process of GA and result with the reduced lead time.

\section{PLANNING SIMULATION WITH THREE-LAYER FRAMEWORK}

To show the validity of the three-layer framework of planning and scheduling, the historical quarterly order data obtained from Huaying Printing Machinery Manufacturing Enterprise, Shantou, China is used for planning simulation with the self-developed PMS. The system is programmed with $\mathrm{C \#}$ under the environment of Microsoft Visual Studio 2010. The database is set up with Microsoft Access 2000 and the query language is SQL.

There are 4 orders for second quarter including 10 customized gravure printing machines. Orders 1 and 2 are permitted a maximum of 7 days tardy, while orders 3 and 4 must be delivered on time. The amount of non-standard parts of ordered products reaches 45,000 , which needs a total 630,000 task hours, whereas the actual shop floor quarterly capacity is only 600,000 man-hours though there are 49 machines with around 850,000 work hours, i.e., the average capacity utilization is about $70 \%$ due to process constraints.
Roughly speaking, the quarterly orders will be delayed for the task exceeding the capacity by $9 \%$. It is true that there was the penalty for the tardiness of one week over the permitted deadline when the production was supervised by the head of the shop floor. The fact is the assembly processes had to be halted because the parts of headstock of gravure had not been machined on time. Compared with the manual planning, the effectiveness of PSM is shown by the following simulation with the proposed three-layer framework.

At the first layer, the resulted quarterly plan of ordered products sequencing with ATC rule is shown in Figure 3, and the detailed value of internal states of OAs are listed as Table I. It is obvious that the upper limit of TTT is exceeded for 3 days due to the serious tardiness of order 1 . Though the tardy phenomenon is not avoided, this result is a bit superior to that of manual planning, and the credit should be given to the application of ATC rule.

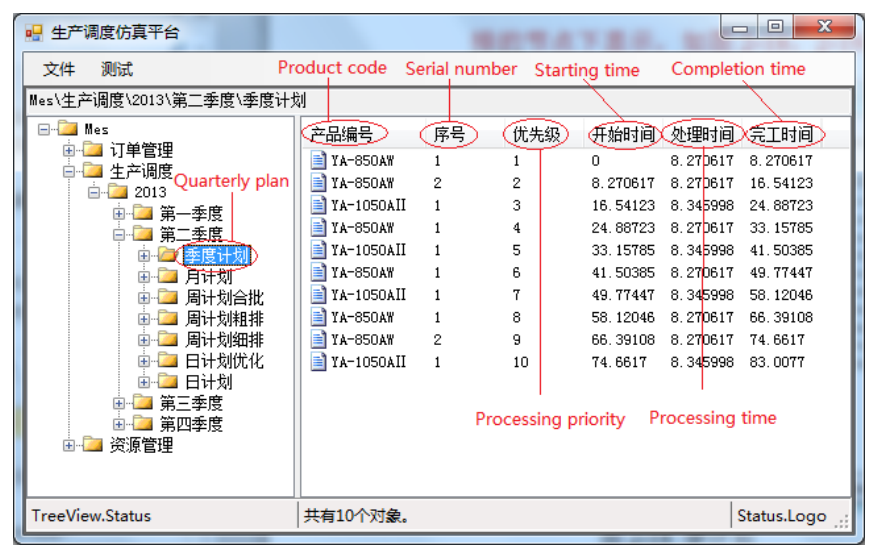

Figure 3. Interface for second quarter planning simulation

The planning scale is further reduced by part batching undertaken at the second layer. When the batching period is selected as the largest 6 , only the resulted weekly plans of June are feasible. Then with the batching period decreased to 5, those of May also become feasible and only April does not. Finally, illustrated as Figure 4, the highest batching size amounts to 116 in the first two weeks plan of April with the batching period chosen as 3 .

Nevertheless, from the data of Table II, the weekly planning makes the TTT reach the worst of 18.625 days with orders 3 and 4 also delayed. The main reason is that there is no sufficient consideration of constraints of machining and assembly processes when tasks divided with TCM rule. The idle-time ignored lengths the completion time and makes the utilization lowered to $67.5 \%$. It should be paid attention that TCM rule is one of the key rules used in manual scheduling. It is this factor that makes the tardy problem severe.

The optimization algorithm GA with the parameter setting shown in Figure 5 is applied for daily planning to solve the problem issued by TCM rule. Moreover, the dynamic reconfiguration of $\mathrm{MU}$ is also implemented in the decoding process to balance machine load and result with the utilization increased to $73 \%$. 


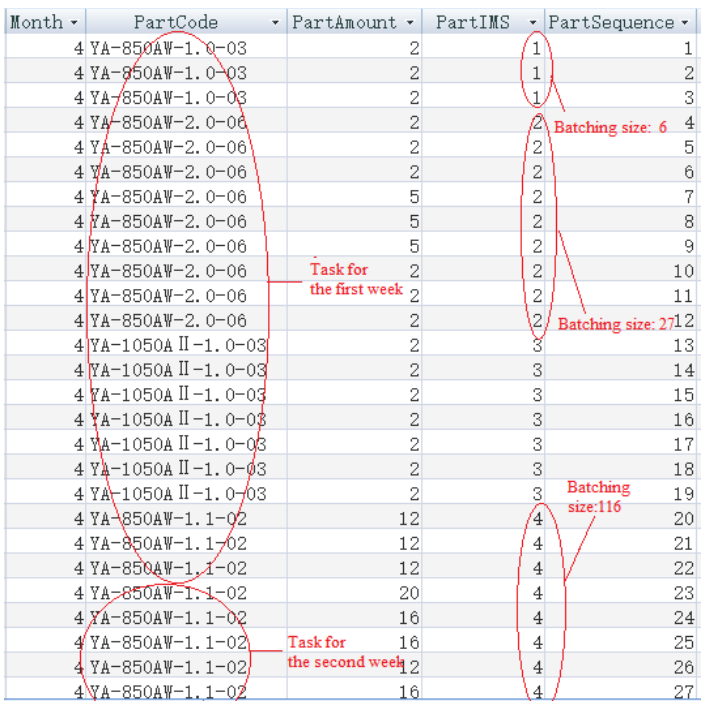

Figure 4. Simulation result of part batching for weekly planning within April of second quarter

Except the individual machining center regarded as a MU, usually two to four kinds of machines constitute one MU with the number of every kind machine ranging from one to four. The operations of shaping and cutting are not commonly used and the number of machines are rather limited. These kinds of bottleneck machines are affiliated with certain MU only at the operation interval decided by RA with STP/WINQ rule. While the machines of general process of turning, milling, and grinding are slightly sufficient, they are also allocated among MU by RA, MA and MUA in the case of heavy load of certain operation within one MU. When applying STP/WINQ rule, the standard of shop balance is defined as the difference of machine load kept within the range of $5 \%$ to $10 \%$.

Through the iterative bottom-up update less than 20 times in the second quarter planning, the checkup TTT finally is reduced below the upper limit with the tardy time of order 1 controlled within the permit while other orders completed a bit early as shown in Table II.

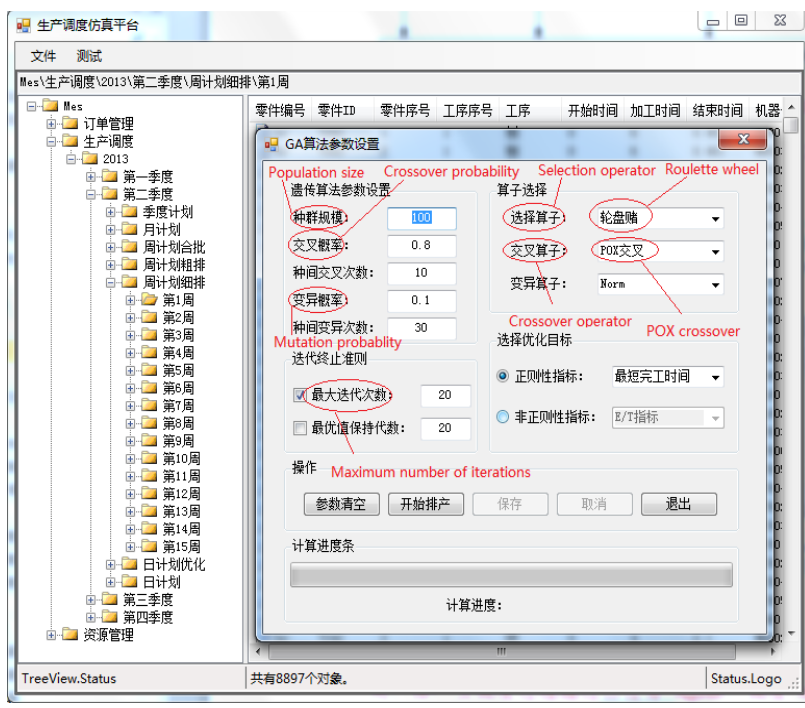

Figure 5. Operator parameter setting of GA for daily planning

\section{CONCLUSION}

To solve the problem of tardiness penalties for small-and-medium-sized packaging machinery enterprises, a large-scale planning and scheduling is considered and a three-layer framework based on multi-agent technique is proposed in this paper to decompose the complex task into subtasks suitable for optimization algorithms. Based on the autonomy of agents and coordination mechanisms for agents, the planning model is developed for each layer by the application of proposed rules and algorithms. The simulation results with the historical data of gravure printing machine orders shows the effectiveness of the three-layer framework controlling the tardy time, thereby reducing the tardy cost. The framework can also handle the dynamic scheduling. The future work will focus on the pattern recognition for various event-driven scheduling to make the system more intelligent and agile.

Table I. Quarterly plan with TTT checkup

\begin{tabular}{|c|c|c|c|c|c|c|c|c|c|}
\hline No. & Product code & DD & MTP & $\mathrm{OU}$ & CL & Weight & Priority & $\mathrm{CT}$ & $\mathrm{TT}$ \\
\hline \multirow{3}{*}{1} & YA-850AW & \multirow{3}{*}{74} & \multirow{3}{*}{7} & \multirow{3}{*}{$\mathrm{D}$} & \multirow{3}{*}{4} & \multirow{3}{*}{3.475} & 8 & \multirow{3}{*}{84} & \multirow{3}{*}{10} \\
\hline & YA-850AW & & & & & & 9 & & \\
\hline & YA-1050A II & & & & & & 10 & & \\
\hline \multirow{3}{*}{2} & YA-850AW & \multirow{3}{*}{34} & \multirow{3}{*}{7} & \multirow{3}{*}{$\mathrm{C}$} & \multirow{3}{*}{3} & \multirow{3}{*}{3.925} & 1 & \multirow{3}{*}{25} & \multirow{3}{*}{0} \\
\hline & YA-850AW & & & & & & 2 & & \\
\hline & YA-1050A II & & & & & & 3 & & \\
\hline \multirow{2}{*}{3} & YA-850AW & \multirow{2}{*}{62} & \multirow{2}{*}{0} & \multirow{2}{*}{ A } & \multirow{2}{*}{1} & \multirow{2}{*}{4.375} & 4 & \multirow{2}{*}{41.5} & \multirow{2}{*}{0} \\
\hline & YA-1050A II & & & & & & 5 & & \\
\hline \multirow{2}{*}{4} & YA-850AW & \multirow{2}{*}{68} & \multirow{2}{*}{0} & \multirow{2}{*}{ B } & \multirow{2}{*}{2} & \multirow{2}{*}{4.025} & 6 & \multirow{2}{*}{58} & \multirow{2}{*}{0} \\
\hline & YA-1050A II & & & & & & 7 & & \\
\hline \multicolumn{9}{|c|}{ TTT } & 10 \\
\hline
\end{tabular}

No.: Order number

DD: due date(day) MTP: Max tardiness permitted
(day)

OU: Order Urgency level, which declines gradually in alphabetical order

CL: Customer level. The larger, the less important

CT: completion time (day)

TT: Tardy Time(day)

MCT: Max. completion time(day)

LN: Lateness(day) 
Table II. TTT checkup based on the plan updated by weekly planning and daily planning

\begin{tabular}{|c|c|c|c|c|c|c|c|c|c|c|c|}
\hline \multirow{2}{*}{ No. } & \multicolumn{5}{|c|}{ Weekly planning } & \multicolumn{6}{|c|}{ Daily planning } \\
\hline & MCT & DD & MTP & TT & TTT & MCT & DD & MTP & TT & $\mathrm{LN}$ & TTT \\
\hline 1 & 87.75 & 74 & 7 & 13.75 & \multirow{4}{*}{18.625} & 80.5 & 74 & 7 & 6.5 & 6.5 & \multirow{4}{*}{6.5} \\
\hline 2 & 69.75 & 68 & 7 & 1.75 & & 64.375 & 68 & 7 & 0 & -3.625 & \\
\hline 3 & 63.75 & 62 & 0 & 1.75 & & 58.5 & 62 & 0 & 0 & -3.5 & \\
\hline 4 & 35.75 & 34 & 0 & 1.375 & & 32.75 & 34 & 0 & 0 & -1.25 & \\
\hline
\end{tabular}

\section{ACKNOWLEDGMENT}

This research was supported by a grant from CSC Foundation.

\section{REFERENCES}

[1] W Shen, D H Norrie. Agent-based systems for intelligent manufacturing: a state-of-the-art survey. Knowledge and Information Systems, 1999, vol.1, no.2, pp.129-156.

[2] W Shen, Q Hao, H J Yoon, D H Norrie. Applications of agent-based systems in intelligent manufacturing: An updated review. Advanced Engineering Informatics, 2006, vol.20, no.4, pp.415-431.

[3] J Kim, S Y Chung, and H J Yoon, "Multi-agent-based scheduling methods for hybrid cellular production lines in semiconductor industry," in Proceedings of The Institution of Mechanical Engineers part B-Journal of Engineering Manufacture, 2014, vol.228, no.12, pp.1701-1702.

[4] H Luo, G Q Huang, Y F Zhang, and Q Y Dai, "Hybrid flow shop scheduling with batch-discrete processors and machine maintenance in time windows," International Journal of Production Research, March 2011, vol.49, no.6, pp.1575-1603.

[5] L C Wang, S K Lin, "A multi-agent based agile manufacturing planning and control system," Computer \& Industrial Engineering, 2009, vol.57, no.2, pp.620-640.

[6] J J Liu, Q X Chen, N Mao and Z A Lin, “A multi-agent-based mould due date setting approach in stochastic production," International Journal of Production Research, March 2011, vol.49, No.5, pp.1353-1371.

[7] S Y Wang and L Wang, "A knowledge-based multi-agent evolutionary algorithm for semiconductor final testing scheduling problem," Knowledge-Based Systems, August 2015, vol.84, No.1, pp.1-9.

[8] A Giret, D Trentesaux, M A Salido, E Garcia, E . A holonic multi-agent methodology to design sustainable intelligent manufacturing control systems. Journal of Cleaner Production, 2017, vol.167, No.11, pp.1370-1386
[9] T Finin, R Frtizon, D Mckay, R McEntire. KQML-A Language and Protocol for Knowledge and Information Exchange. Technical Report, University of Maryland, Baltimore, 1993.

[10] T L Saaty, The Analytic Hierarchy Process [M]. Mc. Graw: Hill International Book Company, 1980.

[11] A P J Vepsalainen, T E Morton. Priority rules for job shops with weighted tardiness costs. Management Science, 1987, vol.33, No.8, pp.1035-1047.

[12] Y H Lee, K Bhaskaran, M Pinedo. A heuristic to minimize the total weighted tardiness with sequence-dependent setups. IIE Transactions, 1997, vol.29, No.1, pp. 45-52.

[13] Y H Wang. Research on the floor shop planning and scheduling for the small-and-medium-sized discrete manufacturing industry. Master thesis, Shantou Univ., China, 2015.

[14] M L Pinedo. Scheduling: theory, algorithms, and systems. 3rd Edition. Springer Science + Business Media, 2008

[15] B Giffler, G L Thompson. Algorithms for solving production-scheduling problems. Operations Research. 1960, vol.8, No.4, pp. 487-503.

[16] C J Zhu, J Cao, Y Hang, C Y Zhang. Applying genetic local search algorithm to solve the job-shop scheduling problem. International Journal of Industrial Engineering. 2012, vol.19, No.9, pp. 341-349.

[17] A Pereira. FMS performance under balancing machine workload and minimizing part movement rules. International Journal of Simulation Modelling, 2011, vol.10, No. 2, pp.91-103.

[18] R W Conway. An experimental investigation of priority assignment in a job shop RM-3789-PR, The Rand Corporation, Santa Monica, Calif., 1964. 